\title{
The effect of spray-drying parameters on the flavor of nonfat dry milk and milk protein concentrate $\mathbf{7 0} \%^{1}$
}

\author{
Curtis W. Park, Mark A. Stout, and MaryAnne Drake ${ }^{2}$ \\ Department of Food, Bioprocessing, and Nutrition Sciences, Southeast Dairy Foods Research Center, North Carolina State University, \\ Raleigh 27695
}

\begin{abstract}
Unit operations during production influence the sensory properties of nonfat dry milk (NFDM) and milk protein concentrate (MPC). Off-flavors in dried dairy ingredients decrease consumer acceptance of ingredient applications. Previous work has shown that spray-drying parameters affect physical and sensory properties of whole milk powder and whey protein concentrate. The objective of this study was to determine the effect of inlet temperature and feed solids concentration on the flavor of NFDM and MPC 70\% (MPC70). Condensed skim milk (50\% solids) and condensed liquid MPC70 (32\% solids) were produced using pilot-scale dairy processing equipment. The condensed products were then spray dried at either 160,210 , or $260^{\circ} \mathrm{C}$ inlet temperature and 30,40 , or $50 \%$ total solids for NFDM and 12,22 , or $32 \%$ for MPC70 in a randomized order. The entire experiment was replicated 3 times. Flavor of the NFDM and MPC70 was evaluated by sensory and instrumental volatile compound analyses. Surface free fat, particle size, and furosine were also analyzed. Both main effects (30, 40, and 50\% solids and 160, 210, and $260^{\circ} \mathrm{C}$ inlet temperature) and interactions between solids concentration and inlet temperature were investigated. Interactions were not significant. In general, results were consistent for NFDM and MPC70. Increasing inlet temperature and feed solids concentration increased sweet aromatic flavor and decreased cardboard flavor and associated lipid oxidation products. Increases in furosine with increased inlet temperature and solids concentration indicated increased Maillard reactions during drying. Particle size increased and surface free fat decreased with increasing inlet temperature and solids concentration. These results demonstrate that

\footnotetext{
Received July 2, 2016.

Accepted August 23, 2016.

${ }^{1}$ Use of names, names of ingredients, and identification of specific

${ }^{2}$ Corresponding author: mdrake@ncsu.edu
} models of equipment is for scientific clarity and does not constitute any endorsement of product by authors, North Carolina State University, or the Southeast Dairy Foods Research Center.
\end{abstract}

increasing inlet temperatures and solids concentration during spray drying decrease off-flavor intensities in NFDM and MPC70 even though the heat treatment is greater compared with low temperature and low solids. Key words: nonfat dry milk, milk protein concentrate, process parameter, flavor

\section{INTRODUCTION}

Dairy powders have developed over the years as a way to extend the shelf life of milk through various drying techniques. Milk powders made from skim milk that contain less than $1.5 \%$ fat (wt/wt) are classified as either nonfat dry milk (NFDM) or skim milk powder (SMP; ADPI, 2015a). Milk retentate, milk permeate, and lactose can be used to adjust protein content to $34 \%$ in SMP but not in NFDM (ADPI, 2015a). Over 1 million $\mathrm{t}$ of SMP and NFDM are used annually as both an ingredient in food applications as well as for direct consumption (USDA, 2015). Milk protein concentrate (MPC) is any concentrated milk product with protein ranging from 40 to $90 \%$ (ADPI, 2015b). The flavor of NFDM and MPC is important because off-flavors negatively affect the consumer acceptance of ingredient applications (Caudle et al., 2005). Flavor variability has been documented in fresh NFDM from different manufacturers (Caudle et al., 2005; Drake et al., 2006) as well as MPC (Drake et al., 2009; Smith et al., 2016a) but currently no published studies have documented the role of unit operations on the flavor of MPC. Current available literature on the flavor of NFDM is limited to flavor properties throughout storage, key aroma compounds, and the effects of off-flavors in ingredient applications (Karagul-Yuceer et al., 2001, 2002; Drake et al., 2003; Caudle et al., 2005; Isleten and Karagul-Yuceer, 2006). Lipid oxidation compounds are the primary source of off flavors in NFDM and MPC stored at $21^{\circ} \mathrm{C}$ (Drake et al., 2006; Smith et al., 2016a).

Typical manufacture of NFDM includes fat separation, pasteurization, evaporation, and spray drying. For MPC, ultrafiltration is performed before concentration to remove the majority of lactose and soluble minerals. Concentration of MPC can be done either by nanofil- 
tration or evaporation. The benefits of using a nanofiltration process include reduced product heating and improved solubility (Cao et al., 2015). Typical solids concentration for condensed skim milk during drying is $50 \%$ whereas for condensed MPC it is lower (around $30 \%$ ). Manufacturers are limited on how much solids can be increased due to the high viscosity of condensed skim milk and condensed MPC (Velez-Ruiz and Barbosa-Canovas, 1998; Bienvenue et al., 2003). Spray drying involves atomization of milk into fine particles, which are mixed with hot air to remove the majority of the water. During drying, the temperature of the individual particles does not generally reach above $60^{\circ} \mathrm{C}$ due to evaporative cooling (Schuck, 2013). The spray-drying process not only affects the water content but also the powder structural and physiochemical characteristics (Vignolles et al., 2007).

Surface free fat (SFF), an important characteristic in dairy powders, is described as fat that is not entirely coated by amphiphilic molecules or protected by a matrix of carbohydrates and proteins during drying (Vignolles et al., 2007). The SFF in whole milk powders can alter important properties of the dried milk powder such as oxidative stability, wettability, dispersibility, solubility, flowability, shelf life, and ability to use in chocolate processing applications (Vignolles et al., 2007). Although NFDM and MPC have a low fat content, lipid oxidation plays a critical role in flavor and flavor stability of NFDM and MPC (Karagul-Yuceer et al., 2002; Caudle et al., 2005; Smith et al., 2016a). Control of SFF through changing processing parameters could potentially affect the flavor and flavor stability of NFDM and MPC (Park and Drake, 2014). Park et al. (2014) recently demonstrated that SFF influenced flavor stability of dried whey proteins and that SFF was affected by spray-drying parameters. Vignolles et al. (2010) also observed that SFF was affected by spray-drying temperatures, with decreasing SFF with increasing inlet temperatures.

Spray-drying parameters affect the flavor of whey protein concentrate (WPC; Park et al., 2014). Decreased off-flavor intensities and corresponding lipid oxidation products were observed with increased inlet temperatures and feed solids concentrations. Increased inlet temperatures may increase the heat treatment given to the dairy powder and decrease nutritional quality. To our knowledge, Maillard reaction products, such as furosine, have not been investigated in dried dairy ingredients spray dried under varying conditions. Given differences in composition and unit operations between WPC, MPC, and NFDM, it is important to investigate the influence of spray-drying parameters on the flavor of NFDM and MPC. The objective of this study was to determine the influence of feed solids concentration and inlet temperature on the flavor of NFDM and MPC 70\% (MPC70).

\section{MATERIALS AND METHODS}

\section{NFDM Production}

Raw bovine skim milk (313 kg) was obtained from the North Carolina State University Dairy Research and Education Unit. The milk was HTST pasteurized at $73^{\circ} \mathrm{C}$ for $16 \mathrm{~s}$ with a plate heat exchanger (model T4 RGS-16/2, SPX Flow Technology, Greensboro, NC) and subsequently cooled to $4^{\circ} \mathrm{C}$ and stored until evaporation $(<6 \mathrm{~h})$. The pasteurized skim milk was evaporated to $50 \%$ solids (wt/wt) in a single stage falling film evaporator. The milk was preheated to $54^{\circ} \mathrm{C}$ and introduced to the evaporator operating at $71^{\circ} \mathrm{C}$ with 75 $\mathrm{kPa}$ vacuum. The condensed milk was then standardized with warm deionized water to either 30,40 , or $50 \%$ solids (wt/wt) and was approximately $50^{\circ} \mathrm{C}$ for $<30$ min before drying. Drying was performed with a spray dryer (model Lab 1, Anhydro Inc., Soeberg, Denmark) with a 2-fluid nozzle with compressed air, operated at an inlet temperature of 160,210 , or $260^{\circ} \mathrm{C}$ with a constant outlet temperature of $90^{\circ} \mathrm{C}$. Outlet temperature was controlled with the feed flow rate into the spray dryer to keep a constant percent moisture in the resulting powders. The TS content in the condensed milk and the spray-dried NFDM was measured using the Smart Turbo moisture/solids analyzer (CEM, Matthews, NC). The order of treatments was completely randomized within $1 \mathrm{~d}$ of production. The entire experiment was replicated 3 times.

\section{MPC70 Production}

Raw bovine skim milk was obtained from the NC State University Dairy Research and Education Unit. The milk was pasteurized as described previously for NFDM production. The pasteurized skim milk was then subjected to UF using a pilot-scale unit (model Lab 46, Filtration Engineering, Champlin, MN). Two spiral-wound membranes were used (nominal cutoff 10 $\mathrm{kDa}$, surface area $6.5 \mathrm{~m}^{2}$ per element; Microdyn, Raleigh, NC) with a recirculation rate of $132 \mathrm{~L} / \mathrm{min}$. The skim milk was heated with a plate heat exchanger to $50^{\circ} \mathrm{C}$ before introduction into the UF unit. Ultrafiltration was performed in 2 stages with concentration factors of $3 \times$ and $4 \times$ in stages 1 and 2, respectively. After stage 1 , diafiltration was performed by adding warm deionized water in an amount equaling $30 \%$ of the original weight of starting skim milk. Solids content of the liquid MPC70 was measured using the Smart Turbo moisture/solids analyzer (CEM). The protein content 
was confirmed using a Sprint Protein Analyzer (CEM) and using an infrared milk analyzer (LactoScope FTIR Advanced, Delta Instruments, Drachten, the Netherlands). The liquid MPC70 was $12 \%$ solids (wt/wt) and $8.4 \%$ protein (wt/wt).

Next, the liquid MPC70 was evaporated to $32 \%$ solids (wt/wt) in the falling film evaporator. The liquid MPC70 was preheated to $54^{\circ} \mathrm{C}$ and introduced to the evaporator operating at $63^{\circ} \mathrm{C}$ with $81 \mathrm{kPa}$ vacuum. The liquid MPC70 was then standardized with warm deionized water to 12,22 , or $32 \%$ solids (wt/wt) and was approximately $50^{\circ} \mathrm{C}$ for $<30$ min before drying. Spray drying was performed as described previously in NFDM manufacture.

\section{Proximate Analysis}

Percent moisture was measured using a vacuum oven (AOAC International, 2000; method 990.20). Fat was measured by ether extraction (AOAC International, 2000; method 932.06).

\section{Descriptive Sensory Analysis}

Sensory testing was done in compliance with the North Carolina State University Institutional Review Board for Human Subjects. Spray-dried NFDM and MPC70 were rehydrated to $10 \%$ solids (wt/vol) in deodorized deionized water. Rehydrated powders were dispensed into soufflé cups (Solo Cup, Highland Park, IL), lidded, and tempered to $21^{\circ} \mathrm{C}$. Rehydrated powders were profiled by a highly trained sensory panel ( $\mathrm{n}=$ 8) each with $>150 \mathrm{~h}$ of training using the Spectrum method with an established dried dairy ingredient lexicon (Drake et al., 2003; Smith et al., 2016b). Panelists expectorated the samples and were given deionized water to cleanse their palates. Compusense Cloud (Compusense, Guelph, Canada) was used for data collection. Each treatment replication was evaluated in duplicate.

\section{Volatile Compound Analysis}

Volatile compounds were extracted using stir bar sorptive extraction (SBSE; Prieto et al., 2010). Each NFDM and MPC70 manufacture replicate was extracted in triplicate. The SBSE was used in place of headspace solid-phase microextraction (SPME) due to the very low concentrations of the volatile compounds in the powders. Stir bar sorptive extraction has a significantly larger surface area of stationary phase to absorb the volatile compounds than SPME. It also has the ability to extract compounds of higher boiling point because the stir bars are immersed in the sample rather than in the headspace. Thermal desorption unit (TDU) tubes were conditioned for $1 \mathrm{~h}$ at $300^{\circ} \mathrm{C}$ before analysis. First, $0.5 \mathrm{~g}$ of powder was dissolved in $5 \mathrm{~mL}$ of HPLC grade water into a $10 \mathrm{~mL}$ amber screw-top vial (Gerstel Inc., Linthicum, MD). Next, $10 \mu \mathrm{L}$ of internal standard was added $(0.81 \mathrm{mg} / \mathrm{kg}$ of 2-methyl-3-heptanone in water; Sigma Aldrich, St. Louis, MO). Sequential stir bar extraction (SSBE) was used with salt addition in the second extraction to more effectively extract compounds of various classes. Extraction of polar compounds is improved with salt, whereas extraction of hydrophobic compounds is decreased with salt (Prieto et al., 2010). One polydimethylsiloxane (PDMS)-coated stir bar (10 $\mathrm{mm} \times 0.5 \mathrm{~mm}$ thickness, Gerstel Inc.) was placed in the vial, sealed, and stirred for $1 \mathrm{~h}$ at $800 \mathrm{rpm}$. After 1 $\mathrm{h}$, the stir bar was briefly rinsed in HPLC-grade water, dried, and placed in a TDU autosampler tube (Gerstel Inc.). Next, $1 \mathrm{~g}$ of $\mathrm{NaCl}$ was added to the sample along with another PDMS stir bar. This stir bar was stirred for $1 \mathrm{~h}$ at $800 \mathrm{rpm}$, rinsed, dried, and placed in the same TDU tube previously mentioned. Compounds were desorbed on to the GC TDU inlet using an autosampler (MPS Autosampler, Gerstel Inc.). The stir bars were desorbed at $250^{\circ} \mathrm{C}$ for $10 \mathrm{~min}$ (TDU, Gerstel Inc.) and the volatile compounds were cryogenically trapped at $-120^{\circ} \mathrm{C}$ (CIS 4 , Gerstel Inc.).

Volatile compounds were analyzed by GC-MS. An Agilent 7890B GC (Agilent Technologies Inc., Santa Clara, CA) with an inert mass selective detector (model 5970A, Agilent) with a ZB-5MS column (30 m $\times 0.25 \mathrm{~mm} \times 0.25 \mu \mathrm{m}$; Phenomenex, Torrance, CA) was used to identify and quantify volatile compounds of interest (Karagul-Yuceer et al., 2001, 2002; Drake et al., 2006). Initial $\mathrm{GC}$ oven conditions were $40^{\circ} \mathrm{C}$ for 3 min with ramp rates of $10^{\circ} \mathrm{C} / \mathrm{min}$ to $90^{\circ} \mathrm{C}, 5^{\circ} \mathrm{C} / \mathrm{min}$ to $200^{\circ} \mathrm{C}$ held for $10 \mathrm{~min}$, and $20^{\circ} \mathrm{C} / \mathrm{min}$ to $250^{\circ} \mathrm{C}$ held for $5 \mathrm{~min}$. Purge time was set to $1.2 \mathrm{~min}$ using helium as the carrier gas at a constant flow rate of $1 \mathrm{~mL} / \mathrm{min}$. Compounds were identified by comparison with the 2014 NIST mass spectral library (NIST, 2014), retention index, and retention time of authentic standards injected under identical conditions. Relative abundance of selected compounds was calculated using recovery of the internal standard.

\section{Surface Free Fat and Particle Size}

Surface free fat was measured by solvent extraction with petroleum ether (GEA Niro Method No. A 10a; GEA, 2005) and was expressed as grams of free fat $/ 100$ $\mathrm{g}$ of total fat. Particle size of the NFDM and MPC70 was measured by laser diffraction (Mastersizer 3000, Malvern, UK). All measurements were performed in duplicate. 


\section{Furosine}

The method of Resmini et al. (1990) was followed to analyze furosine with minor modifications. An amount of NFDM/MPC70 corresponding to 40 to $50 \mathrm{mg}$ of protein was placed in a screw-cap Pyrex tube with a PTFE-lined septa along with $8 \mathrm{~mL}$ of $10.6 \mathrm{M} \mathrm{HCl}$ (Sigma Aldrich). Nitrogen was bubbled through the samples for $2 \mathrm{~min}$ and then capped and heated to $110^{\circ} \mathrm{C}$ for $23 \mathrm{~h}$. After cooling, the hydrolysate was filtered and $0.5 \mathrm{~mL}$ was loaded onto a $500-\mathrm{mg}$ reversed-phase solidphase extraction column (Discovery DSC-18, Supelco) previously conditioned with $5 \mathrm{~mL}$ of methanol and 10 $\mathrm{mL}$ of water. Furosine was eluted with $3 \mathrm{~mL}$ of $3 M$ $\mathrm{HCl}$. Furosine was separated and detected with a Waters HPLC system (Milford, MA). A furosine-dedicated column was used (Altima C8, $250 \times 4.6 \mathrm{~mm}, 5 \mu \mathrm{m}$, Alltech-Grace, Columbia, MD) with isocratic conditions. The mobile phase consisted of $0.4 \%$ acetic acid (Sigma Aldrich) and the column temperature was $35^{\circ} \mathrm{C}$. The UV detection was performed at $280 \mathrm{~nm}$. A 5-point external standard curve ranging from 50 to $400 \mathrm{ng}$ was constructed for quantification of both NFDM and MPC70.

\section{Statistical Analysis}

Two-way ANOVA with means separation with the Fisher's least significant difference post-hoc test was used to analyze the data (version 2015.1, XLStat, Addinsoft, France). Interaction effects between feed solids concentration and inlet temperature were investigated.

\section{RESULTS}

Moisture and fat were not significantly different between treatments $(P>0.05)$ and were $3.12 \pm 0.35 \%$ and $0.651 \pm 0.17 \%$, respectively, for NFDM and $3.98 \pm$ $0.41 \%$ and $1.38 \pm 0.08 \%$ for MPC70, respectively.

The NFDM treatments had distinct flavor profiles (Table 1). Interaction effects between inlet temperature and feed solids concentration for descriptive sensory analysis were not significant $(P>0.05)$. Sweet taste was not affected by feed solids concentration or inlet temperature. A feed solids concentration of 30\% decreased overall aroma intensity compared with $50 \%(P$ $<0.05 ; 1.7$ vs. 1.9$)$. A $30 \%$ feed solids concentration decreased sweet aromatic (1.6 vs. 2.0 vs. 2.1 ) and cooked flavors (3.1 vs. 3.3 vs. 3.3 ) compared with 40 and $50 \%$ solids concentrations, respectively $(P<0.05)$. Decreasing feed solids concentration from 50 to $30 \%$ increased cardboard flavor intensity $(P<0.05$; ND vs. 0.5 vs. 1.1). An inlet temperature of $160^{\circ} \mathrm{C}$ decreased overall aroma (1.7 vs. 1.9 vs. 1.9$)$ and sweet aromatic (1.4 vs. 2.1 vs. 2.2 ) compared with 210 and $260^{\circ} \mathrm{C}$, respectively $(P<0.05)$. Increasing inlet temperature increased cooked flavor intensity (3.1 vs. 3.3 vs. 3.4 ). Cardboard flavor was only detected with an inlet temperature of $160^{\circ} \mathrm{C}$.

The volatile compound profiles of the NFDM treatments were in congruence with the descriptive sensory profiles (Figure 1, Table 2). In general, lipid oxidation products increased with decreasing inlet temperature and feed solids concentration. This is consistent with what has been previously reported in whey protein concentrate 80\% (WPC80; Park et al., 2014). Interaction effects between feed solids concentration and inlet temperature were not significant for the following compounds: furfural, nonanal, furaneol, vanillin, delta-decalactone, delta-dodecalactone, gamma-dodecalactone, butyric acid, hexanoic acid, heptanoic acid, octanoic acid, decanoic acid, dodecanoic acid, methional, 2-acetyl thiazole, phenyl acetaldehyde, and benzaldehyde $(P$ $>0.05)$.

Table 1. Sensory profiles of rehydrated nonfat dry milk spray dried at different feed solids concentrations (30, 40 , or $50 \%)$ and inlet temperatures $\left(160,210 \text {, and } 260^{\circ} \mathrm{C}\right)^{1,2}$

\begin{tabular}{lccccc}
\hline Treatment & $\begin{array}{c}\text { Overall } \\
\text { aroma }\end{array}$ & $\begin{array}{c}\text { Sweet } \\
\text { aromatic }\end{array}$ & Cooked & Cardboard & $\begin{array}{c}\text { Sweet } \\
\text { taste }\end{array}$ \\
\hline $30 \%$ & $1.7^{\mathrm{b}}$ & $1.6^{\mathrm{b}}$ & $3.1^{\mathrm{b}}$ & $1.1^{\mathrm{a}}$ & $2.1^{\mathrm{a}}$ \\
$40 \%$ & $1.8^{\mathrm{ab}}$ & $2.0^{\mathrm{a}}$ & $3.3^{\mathrm{a}}$ & $0.5^{\mathrm{b}}$ & $2.1^{\mathrm{a}}$ \\
$50 \%$ & $1.9^{\mathrm{a}}$ & $2.1^{\mathrm{a}}$ & $3.3^{\mathrm{a}}$ & $\mathrm{ND}^{3}$ & $2.0^{\mathrm{a}}$ \\
& & & & & $2.2^{\mathrm{a}}$ \\
$160^{\circ} \mathrm{C}$ & $1.7^{\mathrm{b}}$ & $1.4^{\mathrm{b}}$ & $3.1^{\mathrm{c}}$ & $\mathrm{ND}$ & $2.1^{\mathrm{a}}$ \\
$210^{\circ} \mathrm{C}$ & $1.9^{\mathrm{a}}$ & $2.1^{\mathrm{a}}$ & $3.3^{\mathrm{b}}$ & $\mathrm{ND}$ & $2.1^{\mathrm{a}}$ \\
$260^{\circ} \mathrm{C}$ & $1.9^{\mathrm{a}}$ & $2.2^{\mathrm{a}}$ & $3.4^{\mathrm{a}}$ & &
\end{tabular}

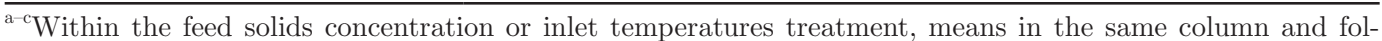
lowed by a different superscript are different $(P<0.05)$.

${ }^{1}$ Interaction effects between solids concentration and inlet temperature were not significant $(P>0.05)$.

${ }^{2}$ Attributes were scored using a 0 - to 15-point universal intensity scale. Most dried dairy ingredient attribute intensities fall between 0 and 4 (Drake et al., 2003).

${ }^{3} \mathrm{ND}=$ not detected 
Both feed solids concentration and inlet temperature significantly affected the volatile compound profile of the rehydrated NFDM. Spray drying at a feed solids concentration of $30 \%$ increased concentrations of furfural and nonanal and decreased delta-decalactone and benzaldehyde compared with 40 or $50 \%(P<0.05)$. When spray dried at $160^{\circ} \mathrm{C}$, a feed solids concentration of 30\% increased hexanal, 2,4-nonadienal, 2,4-decadienal, 1-octen-3-one, and o-aminoacetophenone compared with 40 or $50 \%$ and heptanal compared with $50 \%(P<0.05)$. At an inlet temperature of $210^{\circ} \mathrm{C}$, spray drying at $30 \%$ solids increased concentrations of hexanal, heptanal, maltol, 2,4-nonadienal, 1-octen3 -one, and o-aminoacetophenone compared with 40 or $50 \%$ and 2,4-decadienal compared with 50\% $(P<0.05)$. At an inlet temperature of $210^{\circ} \mathrm{C}$, a solids concentration of $50 \%$ increased delta-nonalactone $(P<0.05)$. When spray dried at $260^{\circ} \mathrm{C}$, a solids concentration of $30 \%$ increased maltol, 2,4-nonadienal, 2,4-decadienal, and o-aminoacetophenone concentrations compared with 40 or $50 \%$ solids $(P<0.05)$.

An inlet temperature of $260^{\circ} \mathrm{C}$ increased the concentration of furfural compared with 160 or $210^{\circ} \mathrm{C}$ and delta-dodecalactone and heptanoic acid compared with $160^{\circ} \mathrm{C}(P<0.05)$. An inlet temperature of $210^{\circ} \mathrm{C}$ decreased nonanal concentration compared with $160^{\circ} \mathrm{C}(P$ $<0.05)$. Spray drying at $160^{\circ} \mathrm{C}$ resulted in decreased butyric acid concentrations compared with 210 or $260^{\circ} \mathrm{C}(P<0.05)$. When dried at $30 \%$ solids, increasing inlet temperature decreased hexanal, 2,4-nonadienal, and 1-octen-3-one concentrations $(P<0.05)$. An inlet temperature of $160^{\circ} \mathrm{C}$ increased decanal, gammadecalactone, and 2,4-decadienal compared with 210 or $260^{\circ} \mathrm{C}$, and $260^{\circ} \mathrm{C}$ decreased heptanal concentration when NFDM was dried at $30 \%$ solids $(P<0.05)$. When dried at $40 \%$ solids, an inlet temperature of $160^{\circ} \mathrm{C}$ increased hexanal, heptanal, decanal, 2,4-nonadienal, and 1-octen-3-one compared with 210 or $260^{\circ} \mathrm{C}$ and 2,4-decadienal compared with $260^{\circ} \mathrm{C}(P<0.05)$. Also, when dried at $40 \%$ solids, maltol concentration increased with a temperature of $260^{\circ} \mathrm{C}$ compared with 160 or $210^{\circ} \mathrm{C}(P<0.05)$. When dried at $50 \%$ solids, an inlet temperature of $160^{\circ} \mathrm{C}$ increased hexanal and 1-octen-3-one concentrations compared with 210 or $260^{\circ} \mathrm{C}$ and heptanal and octanal compared with $210^{\circ} \mathrm{C}(P<$ $0.05)$. At $50 \%$ solids, $210^{\circ} \mathrm{C}$ increased delta-nonalactone and decreased octanal concentrations compared with 160 or $260^{\circ} \mathrm{C}$ and $260^{\circ} \mathrm{C}$ increased maltol concentration compared with 160 or $210^{\circ} \mathrm{C}(P<0.05)$.

As observed in NFDM, inlet temperature and feed solids concentration also affected the sensory and volatile compound profiles of MPC70 $(P<0.05)$. The descriptive sensory profiles are displayed in Table 3 . Interaction effects between solids concentration and inlet

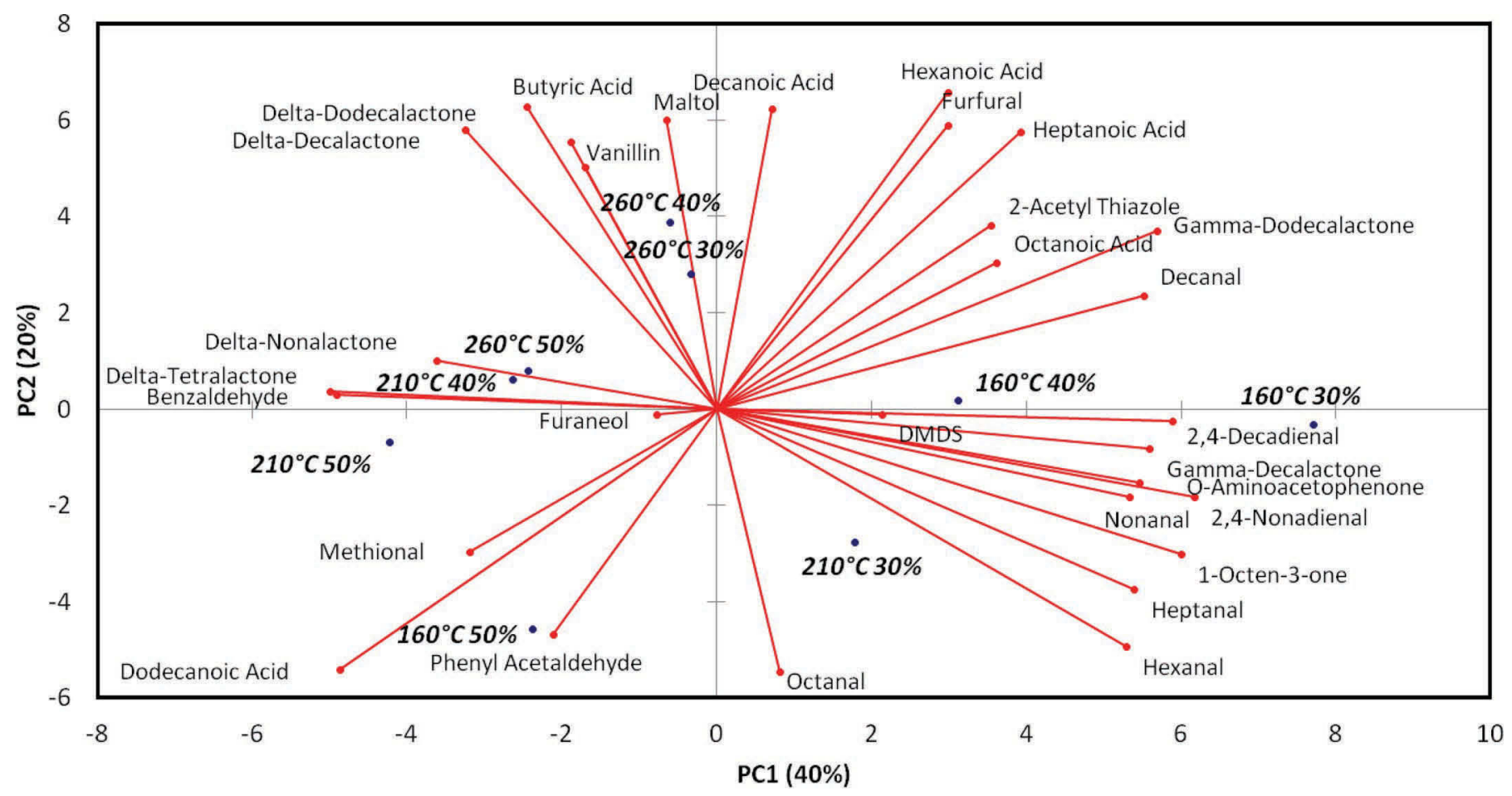

Figure 1. Principal component (PC) biplot of volatile compound analysis of rehydrated nonfat dry milk spray dried at different temperatures and feed solids concentrations. DMDS = dimethyl disulfide. Color version available online. 
Table 2. Volatile compound analysis of rehydrated nonfat dry milk $(\mu \mathrm{g} / \mathrm{kg})$ prepared at different feed solids concentrations $(30,40$, or $50 \%)$ and inlet temperatures $(160,210$, and $\left.260^{\circ} \mathrm{C}\right)$

\begin{tabular}{|c|c|c|c|c|c|c|c|c|c|}
\hline Compound & $160^{\circ} \mathrm{C}, 30 \%$ & $160^{\circ} \mathrm{C}, 40 \%$ & $160^{\circ} \mathrm{C}, 50 \%$ & $210^{\circ} \mathrm{C}, 30 \%$ & $210^{\circ} \mathrm{C}, 40 \%$ & $210^{\circ} \mathrm{C}, 50 \%$ & $260^{\circ} \mathrm{C}, 30 \%$ & $260^{\circ} \mathrm{C}, 40 \%$ & $260^{\circ} \mathrm{C}, 50 \%$ \\
\hline Furfural* & $0.214^{\mathrm{a}}$ & $0.164^{\text {abcd }}$ & $0.137^{\mathrm{d}}$ & $0.185^{\text {abcd }}$ & $0.148^{\text {bcd }}$ & $0.145^{\mathrm{cd}}$ & $0.207^{\mathrm{abc}}$ & $0.222^{\mathrm{a}}$ & $0.208^{\mathrm{ab}}$ \\
\hline Hexanal & $1.41^{\mathrm{a}}$ & $1.07^{\mathrm{b}}$ & $1.050^{\mathrm{b}}$ & $1.03^{\mathrm{b}}$ & $0.654^{\mathrm{c}}$ & $0.655^{\mathrm{c}}$ & $0.593^{\mathrm{c}}$ & $0.674^{\mathrm{c}}$ & $0.654^{\mathrm{c}}$ \\
\hline Heptanal & $0.654^{\mathrm{ab}}$ & $0.674^{\mathrm{a}}$ & $0.551^{\mathrm{bc}}$ & $0.644^{\mathrm{ab}}$ & $0.418^{\text {cd }}$ & $0.389^{\mathrm{d}}$ & $0.470^{\text {cd }}$ & $0.458^{\mathrm{cd}}$ & $0.435^{\mathrm{cd}}$ \\
\hline Octanal & $0.396^{\mathrm{abc}}$ & $0.371^{\mathrm{bc}}$ & $0.465^{\mathrm{a}}$ & $0.393^{\mathrm{abc}}$ & $0.319^{c}$ & $0.324^{\mathrm{c}}$ & $0.332^{\mathrm{c}}$ & $0.350^{\mathrm{c}}$ & $0.443^{\mathrm{ab}}$ \\
\hline Nonanal* & $4.86^{\mathrm{a}}$ & $3.81^{\text {cd }}$ & $3.73^{\text {cde }}$ & $4.50^{\mathrm{ab}}$ & $3.40^{\mathrm{de}}$ & $3.14^{\mathrm{e}}$ & $4.13^{\mathrm{bc}}$ & $3.43^{\mathrm{de}}$ & $3.85^{\mathrm{cd}}$ \\
\hline Decanal & $1.06^{\mathrm{a}}$ & $0.927^{\mathrm{ab}}$ & $0.576^{\text {cde }}$ & $0.625^{\text {cde }}$ & $0.517^{\mathrm{de}}$ & $0.446^{\mathrm{e}}$ & $0.765^{\mathrm{bc}}$ & $0.707^{\text {cd }}$ & $0.788^{\mathrm{bc}}$ \\
\hline Maltol & $0.181^{\mathrm{d}}$ & $0.168^{\mathrm{d}}$ & $0.114^{\mathrm{d}}$ & $0.289^{\mathrm{c}}$ & $0.164^{\mathrm{d}}$ & $0.178^{\mathrm{d}}$ & $0.614^{\mathrm{a}}$ & $0.382^{\mathrm{b}}$ & $0.424^{\mathrm{b}}$ \\
\hline Furaneol* & $0.018^{\mathrm{a}}$ & $0.017^{\mathrm{a}}$ & $0.017^{\mathrm{a}}$ & $0.022^{\mathrm{a}}$ & $0.016^{\mathrm{a}}$ & $0.024^{\mathrm{a}}$ & $0.025^{\mathrm{a}}$ & $0.016^{\mathrm{a}}$ & $0.017^{\mathrm{a}}$ \\
\hline Vanillin* & $0.019^{\mathrm{b}}$ & $0.017^{\mathrm{b}}$ & $0.013^{\mathrm{b}}$ & $0.018^{\mathrm{b}}$ & $0.033^{\mathrm{a}}$ & $0.019^{\mathrm{b}}$ & $0.022^{\mathrm{ab}}$ & $0.022^{\mathrm{ab}}$ & $0.023^{\mathrm{ab}}$ \\
\hline Delta-nonalactone & $0.113^{\mathrm{c}}$ & $0.135^{\mathrm{b}}$ & $0.122^{\text {bc }}$ & $0.131^{\mathrm{bc}}$ & $0.129^{\mathrm{bc}}$ & $0.181^{\mathrm{a}}$ & $0.131^{\mathrm{b}}$ & $0.139^{\mathrm{b}}$ & $0.130^{\text {be }}$ \\
\hline Delta-decalactone* & $1.05^{\mathrm{bc}}$ & $1.21^{\mathrm{ab}}$ & $1.17^{\mathrm{abc}}$ & $0.954^{\mathrm{c}}$ & $1.21^{\mathrm{ab}}$ & $1.20^{\mathrm{ab}}$ & $1.21^{\mathrm{ab}}$ & $1.33^{\mathrm{a}}$ & $1.18^{\mathrm{abc}}$ \\
\hline Delta-dodecalactone* & $0.292^{\text {bc }}$ & $0.287^{\mathrm{bc}}$ & $0.260^{c}$ & $0.259^{\mathrm{c}}$ & $0.280^{\mathrm{bc}}$ & $0.371^{\mathrm{a}}$ & $0.339^{\mathrm{ab}}$ & $0.354^{\mathrm{ab}}$ & $0.341^{\mathrm{ab}}$ \\
\hline Delta-tetralactone & $0.096^{\text {de }}$ & $0.091^{\mathrm{e}}$ & $0.148^{\mathrm{ab}}$ & $0.117^{\text {cde }}$ & $0.132^{\mathrm{bc}}$ & $0.133^{\mathrm{bc}}$ & $0.120^{\text {cd }}$ & $0.144^{\mathrm{bc}}$ & $0.173^{\mathrm{a}}$ \\
\hline Gamma-decalactone & $0.213^{\mathrm{a}}$ & $0.165^{\mathrm{b}}$ & $0.122^{\mathrm{de}}$ & $0.152^{\mathrm{bc}}$ & $0.130^{\text {cde }}$ & $0.148^{\mathrm{bcd}}$ & $0.144^{\text {bcde }}$ & $0.121^{\mathrm{e}}$ & $0.128^{\text {cde }}$ \\
\hline Gamma-dodecalactone* & $0.061^{\mathrm{a}}$ & $0.053^{\mathrm{a}}$ & $0.044^{\mathrm{a}}$ & $0.049^{\mathrm{a}}$ & $0.048^{\mathrm{a}}$ & $0.042^{\mathrm{a}}$ & $0.050^{\mathrm{a}}$ & $0.054^{\mathrm{a}}$ & $0.049^{\mathrm{a}}$ \\
\hline 2,4-Nonadienal & $0.034^{\mathrm{a}}$ & $0.024^{\mathrm{b}}$ & $0.017^{\mathrm{cd}}$ & $0.024^{\mathrm{b}}$ & $0.016^{\text {cd }}$ & $0.014^{\mathrm{d}}$ & $0.020^{\mathrm{c}}$ & $0.014^{\mathrm{d}}$ & $0.015^{\mathrm{d}}$ \\
\hline 2,4-Decadienal & $0.076^{\mathrm{a}}$ & $0.051^{\mathrm{b}}$ & $0.041^{\mathrm{c}}$ & $0.051^{\mathrm{b}}$ & $0.045^{\mathrm{bc}}$ & $0.041^{\mathrm{c}}$ & $0.052^{\mathrm{b}}$ & $0.041^{\mathrm{c}}$ & $0.037^{\mathrm{c}}$ \\
\hline Butyric acid* & $0.613^{\mathrm{cd}}$ & $0.608^{\mathrm{cd}}$ & $0.537^{\mathrm{d}}$ & $0.923^{\mathrm{b}}$ & $1.01^{\mathrm{ab}}$ & $0.769^{\mathrm{bc}}$ & $1.17^{\mathrm{a}}$ & $0.932^{\mathrm{b}}$ & $0.796^{\mathrm{bc}}$ \\
\hline Hexanoic acid* & $1.70^{\mathrm{ab}}$ & $1.66^{\mathrm{ab}}$ & $1.24^{\mathrm{c}}$ & $1.36^{\mathrm{bc}}$ & $1.50^{\mathrm{abc}}$ & $1.54^{\mathrm{abc}}$ & $1.78^{\mathrm{a}}$ & $1.61^{\mathrm{abc}}$ & $1.37^{\mathrm{bc}}$ \\
\hline Heptanoic acid* & $0.200^{\mathrm{ab}}$ & $0.187^{\mathrm{abc}}$ & $0.157^{\text {cd }}$ & $0.173^{\mathrm{bcd}}$ & $0.172^{\mathrm{bcd}}$ & $0.148^{\mathrm{d}}$ & $0.178^{\text {abcd }}$ & $0.209^{\mathrm{a}}$ & $0.185^{\mathrm{abc}}$ \\
\hline Octanoic acid* & $3.34^{\mathrm{ab}}$ & $3.81^{\mathrm{a}}$ & $2.83^{\mathrm{d}}$ & $2.83^{\text {cd }}$ & $2.92^{\mathrm{bcd}}$ & $2.95^{\text {bcd }}$ & $2.89^{\mathrm{bcd}}$ & $3.30^{\mathrm{bc}}$ & $3.08^{\mathrm{bcd}}$ \\
\hline Decanoic acid* & $2.16^{\text {bc }}$ & $2.55^{\mathrm{ab}}$ & $1.91^{\mathrm{c}}$ & $1.89^{\mathrm{c}}$ & $2.036^{\mathrm{c}}$ & $1.93^{\mathrm{c}}$ & $2.29^{\mathrm{abc}}$ & $2.54^{\mathrm{ab}}$ & $2.711^{\mathrm{a}}$ \\
\hline Dodecanoic acid* & $1.30^{\mathrm{b}}$ & $1.50^{\mathrm{ab}}$ & $1.81^{\mathrm{a}}$ & $1.64^{\mathrm{ab}}$ & $1.65^{\mathrm{ab}}$ & $1.77^{\mathrm{a}}$ & $1.38^{\mathrm{b}}$ & $1.52^{\mathrm{ab}}$ & $1.60^{\mathrm{ab}}$ \\
\hline 1-Octen-3-one & $0.150^{\mathrm{a}}$ & $0.106^{\mathrm{b}}$ & $0.067^{\mathrm{d}}$ & $0.087^{\mathrm{c}}$ & $0.044^{\mathrm{e}}$ & $0.033^{\mathrm{e}}$ & $0.040^{\mathrm{e}}$ & $0.041^{\mathrm{e}}$ & $0.032^{\mathrm{e}}$ \\
\hline Methional* & $0.003^{\mathrm{b}}$ & $0.003^{\mathrm{ab}}$ & $0.005^{\mathrm{a}}$ & $0.003^{\mathrm{ab}}$ & $0.003^{\text {ab }}$ & $0.004^{\mathrm{ab}}$ & $0.003^{\mathrm{ab}}$ & $0.003^{\mathrm{b}}$ & $0.006^{\mathrm{a}}$ \\
\hline 2-Acetyl thiazole* & $0.004^{\mathrm{abc}}$ & $0.004^{\mathrm{abc}}$ & $0.002^{\mathrm{d}}$ & $0.004^{\mathrm{a}}$ & $0.004^{\mathrm{ab}}$ & $0.003^{\mathrm{cd}}$ & $0.003^{\mathrm{bcd}}$ & $0.004^{\mathrm{a}}$ & $0.003^{\mathrm{abc}}$ \\
\hline O-Aminoacetophenone & $0.015^{\mathrm{a}}$ & $0.012^{\mathrm{b}}$ & $0.006^{\text {def }}$ & $0.016^{\mathrm{a}}$ & $0.008^{\mathrm{cd}}$ & $0.005^{\text {ef }}$ & $0.007^{\text {de }}$ & $0.009^{\mathrm{bc}}$ & $0.004^{\mathrm{f}}$ \\
\hline Phenyl acetaldehyde* & $0.119^{\mathrm{a}}$ & $0.135^{\mathrm{a}}$ & $0.163^{\mathrm{a}}$ & $0.229^{\mathrm{a}}$ & $0.159^{\mathrm{a}}$ & $0.181^{\mathrm{a}}$ & $0.173^{\mathrm{a}}$ & $0.091^{\mathrm{a}}$ & $0.186^{\mathrm{a}}$ \\
\hline Dimethyl disulfide & $0.235^{\mathrm{a}}$ & $0.216^{\mathrm{ab}}$ & $0.212^{\mathrm{ab}}$ & $0.217^{\text {ab }}$ & $0.190^{\mathrm{b}}$ & $0.229^{\mathrm{a}}$ & $0.195^{\mathrm{b}}$ & $0.233^{\mathrm{a}}$ & $0.222^{\mathrm{ab}}$ \\
\hline Benzaldehyde* & $0.700^{\mathrm{b}}$ & $0.856^{\mathrm{ab}}$ & $0.872^{\mathrm{ab}}$ & $0.760^{\mathrm{b}}$ & $0.966^{\mathrm{a}}$ & $0.876^{\mathrm{ab}}$ & $0.789^{\mathrm{b}}$ & $0.845^{\mathrm{ab}}$ & $0.854^{\mathrm{ab}}$ \\
\hline
\end{tabular}

*Interaction effects between solids concentration and inlet temperature were not significant for compounds marked with an asterisk $(P>0.05)$. 
Table 3. Sensory profiles of rehydrated milk protein concentrate $70 \%$ (MPC70) spray dried at different inlet temperatures $\left(160,210\right.$, and $\left.260^{\circ} \mathrm{C}\right)$ and feed solids concentrations $(30,40 \text {, or } 50 \%)^{1}$

\begin{tabular}{lccccc}
\hline Treatment & $\begin{array}{c}\text { Aroma } \\
\text { intensity }\end{array}$ & $\begin{array}{c}\text { Sweet } \\
\text { aromatic }\end{array}$ & Cooked & Cardboard & Fatty \\
\hline $160^{\circ} \mathrm{C}$ & $1.6^{\mathrm{a}}$ & $0.9^{\mathrm{c}}$ & $2.0^{\mathrm{a}}$ & $1.8^{\mathrm{a}}$ & $1.1^{\mathrm{a}}$ \\
$210^{\circ} \mathrm{C}$ & $1.6^{\mathrm{a}}$ & $1.1^{\mathrm{b}}$ & $2.0^{\mathrm{a}}$ & $1.5^{\mathrm{b}}$ & $\mathrm{ND}^{3}$ \\
$260^{\circ} \mathrm{C}$ & $1.5^{\mathrm{a}}$ & $1.4^{\mathrm{a}}$ & $2.0^{\mathrm{a}}$ & $1.3^{\mathrm{b}}$ & $\mathrm{ND}$ \\
$12 \%$ & & & & & $1.7^{\mathrm{a}}$ \\
$22 \%$ & $1.5^{\mathrm{a}}$ & $0.8^{\mathrm{b}}$ & $2.0^{\mathrm{a}}$ & $1.4^{\mathrm{a}}$ & $\mathrm{ND}^{\mathrm{a}}$ \\
$32 \%$ & $1.6^{\mathrm{a}}$ & $1.2^{\mathrm{a}}$ & $2.0^{\mathrm{a}}$ & $1.3^{\mathrm{b}}$ & $\mathrm{ND}$ \\
\hline
\end{tabular}

${ }^{a-c}$ Within the inlet temperatures or feed solids concentration treatment, means in the same column followed by a different letter are different $(P<0.05)$.

${ }^{1}$ Interaction effects between solids concentration and inlet temperature were not significant $(P>0.05)$.

${ }^{2}$ Attributes were scored using a 0 - to 15 -point universal intensity scale. Most dried dairy ingredient attribute intensities fall between 0 and 4 (Drake et al., 2003).

${ }^{3} \mathrm{ND}=$ not detected

temperature were not significant $(P>0.05)$. Aroma intensity and cooked flavor were not affected by solids concentration or inlet temperature $(P>0.05)$. A solids concentration of $12 \%$ decreased sweet aromatic flavor compared with 22 or $32 \%$ and increased cardboard flavor compared with $32 \%(P<0.05)$. Fatty flavor was only detected in MPC70 spray dried at $12 \%$ solids or $160^{\circ} \mathrm{C}$. Increasing inlet temperature increased sweet aromatic flavor $(P<0.05)$. Cardboard flavor intensity increased when MPC70 was spray dried at $160^{\circ} \mathrm{C}$ compared with 210 or $260^{\circ} \mathrm{C}(P<0.05)$.

Volatile compound profiles of rehydrated MPC70 followed similar trends as in NFDM (Figure 2; Table 4). In general, lipid oxidation products increased with decreasing inlet temperature and feed solids concentration. Interaction effects were not significant for furfuryl alcohol, furfural, maltol, furaneol, delta-nonalactone, delta-decalactone, delta-dodecalactone, dodecanoic acid, phenyl acetaldehyde, or dimethyl disulfide $(P>$ 0.05). An inlet temperature of $260^{\circ} \mathrm{C}$ increased furfuryl alcohol and maltol concentrations compared with $160^{\circ} \mathrm{C}$ $(P<0.05)$. A solids concentration of $32 \%$ during spray drying increased furfuryl alcohol, delta-dodecalactone, and dodecanoic acid concentrations compared with $12 \%(P<0.05)$.

When MPC70 was spray dried at $160^{\circ} \mathrm{C}$, a solids concentration of $12 \%$ increased hexanal, decanal, 2,4-nonadienal, and 2,4-decadienal compared with 22 or $32 \%$ and gamma-decalactone compared with $22 \%(P<0.05)$. At $160^{\circ} \mathrm{C}, 32 \%$ solids increased butyric acid, heptanoic acid, decanoic acid, 2-acetyl thiazole, and benzaldehyde compared with 12 or $22 \%$ solids and heptanal, octanal, nonanal, and hexanoic acid compared with $22 \%$ solids $(P<0.05)$. At an inlet temperature of $210^{\circ} \mathrm{C}$, increasing solids decreased hexanal and octanal concentrations $(P<0.05)$. At $210^{\circ} \mathrm{C}, 12 \%$ solids increased heptanal, nonanal, and o-aminoacetophenone and decreased delta tetralactone compared with 22 or $32 \%$ solids $(P$ $<0.05)$. When dried at $210^{\circ} \mathrm{C}$, a solids concentration of $32 \%$ increased vanillin, gamma-dodecalactone, butyric acid, hexanoic acid, heptanoic acid, and 2-acetyl thiazole compared with 12 or $22 \%$ solids $(P<0.05)$. When dried at $260^{\circ} \mathrm{C}$, increasing solids concentration decreased hexanal concentration $(P<0.05)$. In addition, at $260^{\circ} \mathrm{C}, 12 \%$ solids increased heptanal, 1-octen3 -one, and o-aminoacetophenone compared with 22 or $32 \%$ solids and octanal and 2,4-nonadienal compared with $22 \%$ solids $(P<0.05)$. At an inlet temperature of $260^{\circ} \mathrm{C}, 32 \%$ solids increased vanillin, octanoic acid, and 2-acetyl thiazole compared with 12 or $22 \%$ solids and decreased nonanal compared with $12 \%$ solids $(P$ $<0.05$ ). When MPC70 was dried at $12 \%$ solids and an inlet temperature of $160^{\circ} \mathrm{C}$ increased hexanal, heptanal, nonanal, decanal, 2,4-decadienal, and o-aminoacetophenone concentrations were observed compared with 210 or $260^{\circ} \mathrm{C}(P<0.05)$. At $22 \%$ solids, drying at $160^{\circ} \mathrm{C}$ increased hexanal, heptanal, octanal, and nonanal compared with 210 or $260^{\circ} \mathrm{C}$ and drying at $260^{\circ} \mathrm{C}$ increased butyric acid, heptanoic acid, and 2-acetyl thiazole compared with 160 or $210^{\circ} \mathrm{C}(P<0.05)$. When spray dried at $32 \%$ solids, an inlet temperature of $160^{\circ} \mathrm{C}$ increased hexanal, heptanal, octanal, and nonanal compared with 210 or $260^{\circ} \mathrm{C}$ and an inlet temperature of $260^{\circ} \mathrm{C}$ increased gamma-decalactone and 2-acetyl thiazole compared with $160^{\circ} \mathrm{C}(P<0.05)$.

Furosine (FUR) concentration was also affected by spray-drying parameters in both NFDM and MPC70 (Figures 3 and 4). Furosine is an indicator of early Maillard reactions and has been used to measure nutritional quality of dairy proteins as it measures the amount of blocked lysine (Mehta and Deeth, 2015). Interaction effects between inlet temperature and feed solids concentration were not significant for furosine content in both NFDM and MPC70 $(P>0.05)$. In NFDM, an 
inlet temperature of $260^{\circ} \mathrm{C}$ increased FUR concentration compared with 160 or $210^{\circ} \mathrm{C}$ ( 20.3 vs. 13.9 vs. 15.2 mg of FUR/100 g of protein; $P<0.05$ ). Also, a feed solids concentration of $30 \%$ decreased FUR compared with 40 or $50 \%$ (12.3 vs. 17.9 vs. $19.3 \mathrm{mg}$ of FUR/100 $\mathrm{g}$ of protein; $P<0.05)$. Similarly, in MPC70 an inlet temperature of $260^{\circ} \mathrm{C}$ increased FUR concentrations compared with 160 or $210^{\circ} \mathrm{C}(15.9$ vs. 9.5 vs. $13.4 \mathrm{mg}$ of FUR/100 g of protein; $P<0.05)$. Solids concentration did not affect FUR concentration in MPC70 $(P>$ 0.05).

Particle size and SFF were also different between treatments (Table 5, Figure 5). Interaction effects between temperature and solids concentration were not significant for either particle size or SFF in NFDM and MPC70 $(P>0.05)$. In NFDM, both inlet temperature and solids concentration affected particle size $(P$ $<0.05)$ but neither affected SFF $(P>0.05)$. Increasing inlet temperature increased both the Dx90 (90\% of particle diameters below that value) (77.6 vs. 97.8 vs. $105 \mu \mathrm{m}$ ) and the $\mathrm{D}[4,3]$ (volumetric mean; 35.1 vs. 45.8 vs. $62.5 \mu \mathrm{m} ; P<0.05$ ). Similarly, increasing solids concentration increased Dx90 (77.4 vs. 85.8 vs. 118 $\mu \mathrm{m})$ and $\mathrm{D}[4,3]$ (35.3 vs. 40.1 vs. $68.0 \mu \mathrm{m})$. In MPC70, increasing inlet temperature increased $\mathrm{D}[4,3]$ (31.4 vs. 43.5 vs. $49.2 \mu \mathrm{m})$ and $160^{\circ} \mathrm{C}$ decreased Dx90 compared with 210 or $260^{\circ} \mathrm{C}(72.7$ vs. 93.3 vs. $99.5 \mu \mathrm{m} ; P<$ 0.05). The specific surface area increased in MPC70 with decreasing inlet temperature (1,850 vs. 2,180 vs. $\left.3,080 \mathrm{~m}^{2} / \mathrm{kg} ; P<0.05\right)$, which is consistent with the smaller particle size at lower inlet temperatures. Particle size of MPC70 spray dried at $12 \%$ decreased compared with 22 or $32 \%$ as observed in Dx90 (76.9 vs. 96.9 vs. $91.7 \mu \mathrm{m}), \mathrm{D}[4,3]$ (34.2 vs. 46.8 vs. $43.1 \mu \mathrm{m})$, and specific surface area $\left(2,600\right.$ vs. 2,140 vs. $2,300 \mathrm{~m}^{2} /$ $\mathrm{kg} ; P<0.05)$. The SFF increased in MPC70 spray dried at $160^{\circ} \mathrm{C}$ compared with 210 or $260^{\circ} \mathrm{C}$ (0.036 vs. 0.027 vs. $0.022 \mathrm{~g}$ of $\mathrm{SFF} / 100 \mathrm{~g}$ of MPC70) and at $12 \%$ compared with 22 or $32 \%$ (0.046 vs. 0.020 vs. $0.020 \mathrm{~g}$ of $\mathrm{SFF} / 100 \mathrm{~g}$ of MPC70; $P<0.05)$. The increase in SFF was most likely due to the increase in surface area due to the smaller particle size.

\section{DISCUSSION}

The flavors of NFDM and MPC70 are distinct even though many of the same volatile compounds are responsible for their flavor (Karagul-Yuceer et al., 2001,

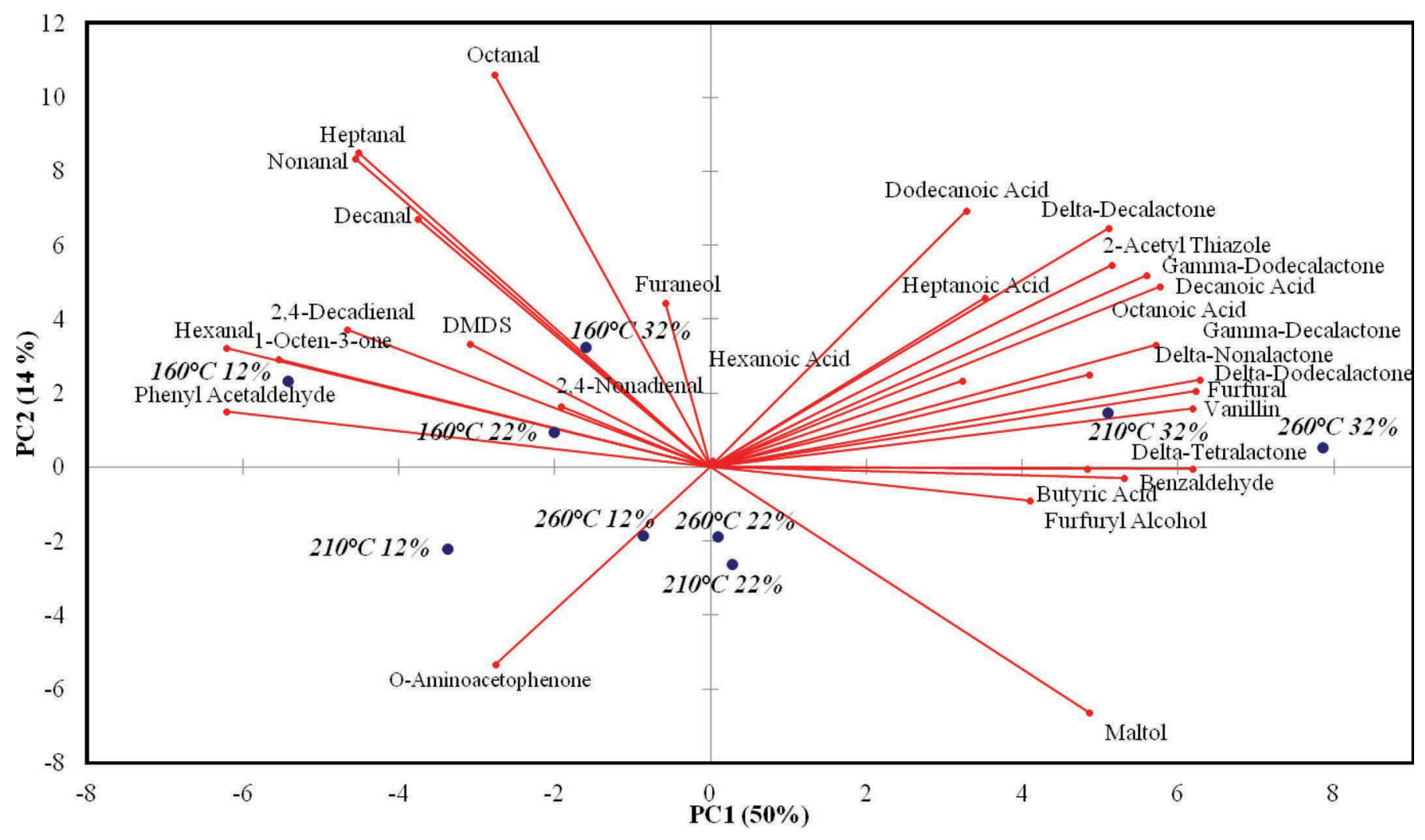

Figure 2. Principal component (PC) biplot of volatile compound analysis of rehydrated milk protein concentrate $70 \%$ (MPC70) spray dried under various conditions. DMDS = dimethyl disulfide. Color version available online. 
PARK ET AL.

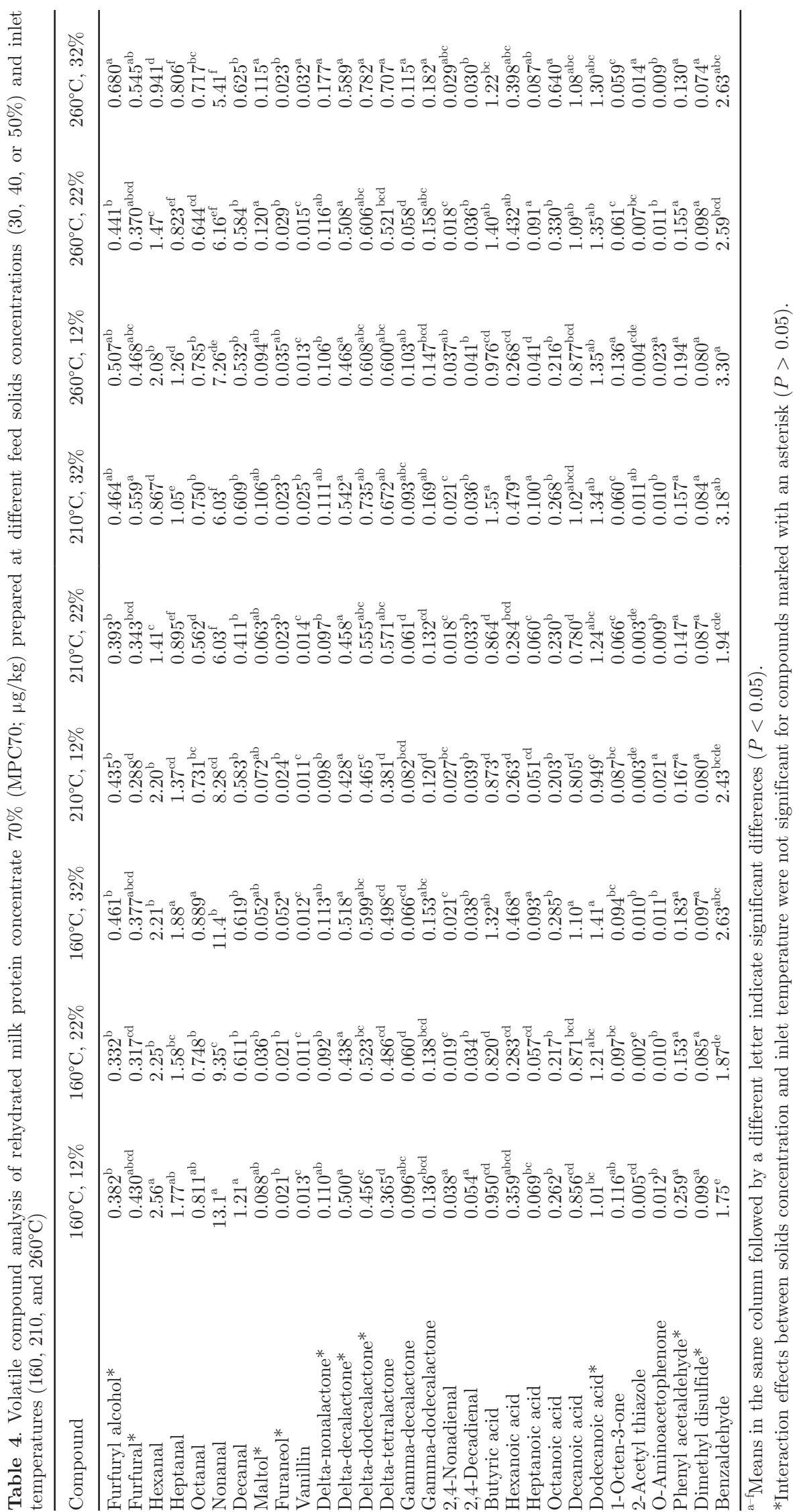




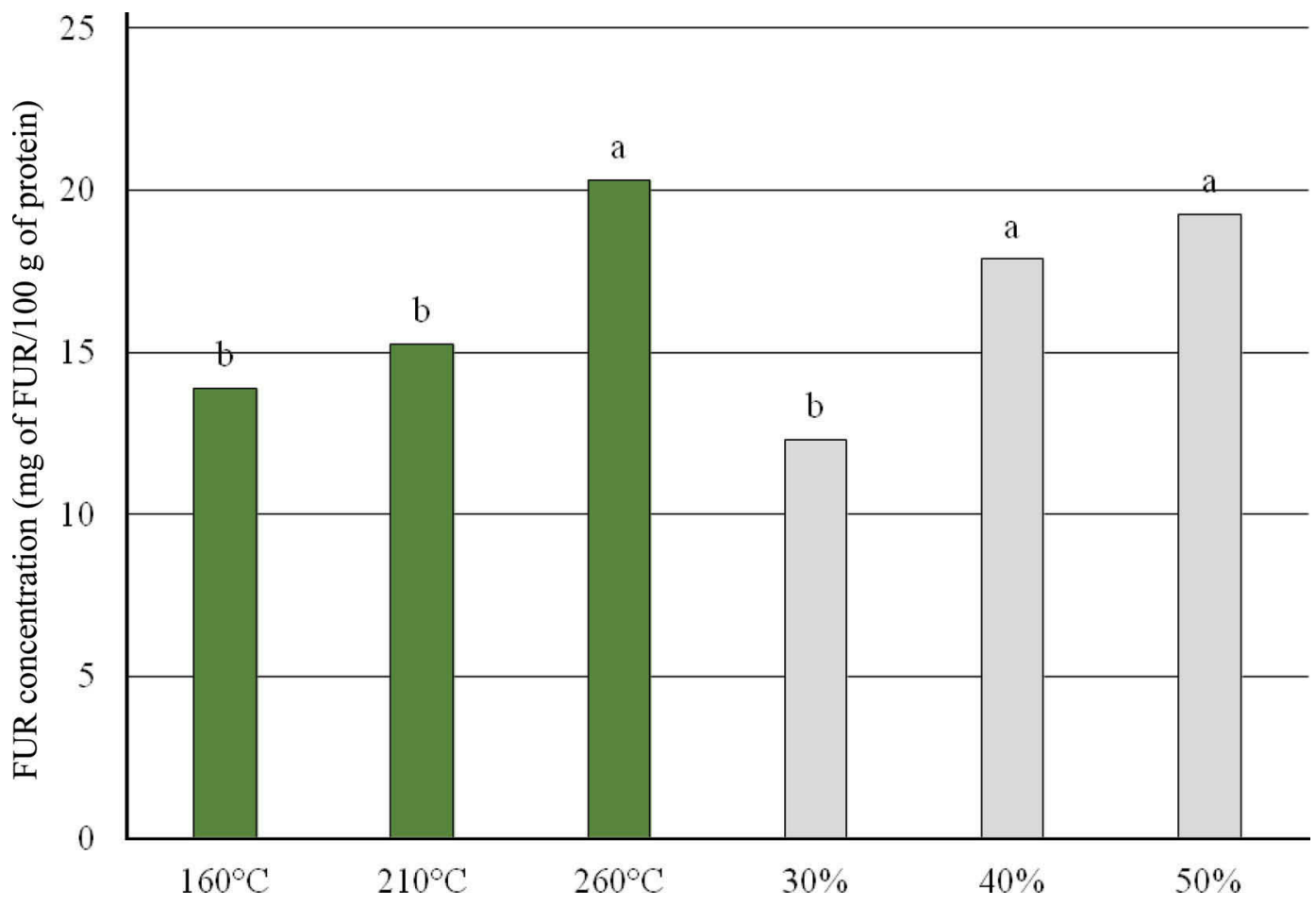

Figure 3. Furosine (FUR) concentration (mg/100 g of protein) in spray-dried nonfat dry milk (NFDM). Bars with the same shading and different lettering indicate a significant difference $(P<0.05)$. Interaction effects between solids concentration and inlet temperature were not significant $(P>0.05)$. Color version available online.

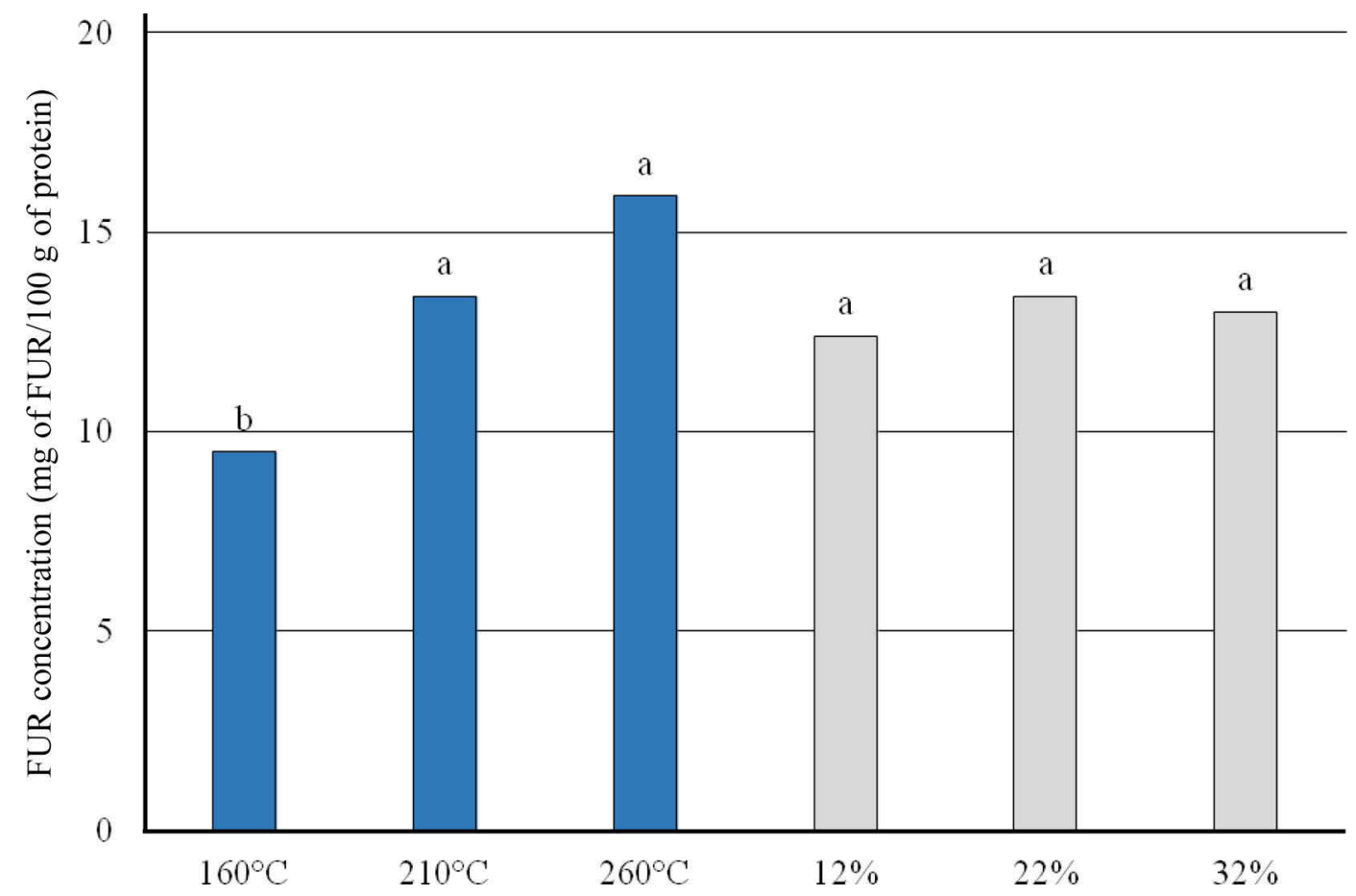

Figure 4. Furosine (FUR) concentration (mg/100 g of protein) in spray-dried milk protein concentrate $70 \%$ (MPC70). Bars with the same shading and different lettering indicate a significant difference $(P<0.05)$. Interaction effects between solids concentration and inlet temperature were not significant $(P>0.05)$. Color version available online. 
Table 5. Surface free fat, particle size, ${ }^{1}$ and specific surface area of nonfat dry milk (NFDM) and milk protein concentrate $70 \%$ (MPC70) powders prepared at different feed solids concentrations and inlet temperatures ${ }^{2}$

\begin{tabular}{|c|c|c|c|c|c|c|c|}
\hline \multirow[b]{2}{*}{ Item } & \multicolumn{3}{|c|}{ NFDM } & \multicolumn{4}{|c|}{ MPC70 } \\
\hline & $\begin{array}{c}\text { Surface free } \\
\text { fat }(\mathrm{g} / 100 \mathrm{~g})\end{array}$ & $\begin{array}{l}\text { Dx90 } \\
(\mu \mathrm{m})\end{array}$ & $\begin{array}{c}\mathrm{D}[4,3] \\
(\mu \mathrm{m})\end{array}$ & $\begin{array}{c}\text { Surface free } \\
\text { fat }(\mathrm{g} / 100 \mathrm{~g})\end{array}$ & $\begin{array}{l}\text { Dx90 } \\
(\mu \mathrm{m})\end{array}$ & $\begin{array}{c}\mathrm{D}[4,3] \\
(\mu \mathrm{m})\end{array}$ & $\begin{array}{l}\text { Specific surface } \\
\text { area }\left(\mathrm{m}^{2} / \mathrm{kg}\right)\end{array}$ \\
\hline \multicolumn{8}{|c|}{ Inlet temperature $\left({ }^{\circ} \mathrm{C}\right)$} \\
\hline 160 & $0.011^{\mathrm{a}}$ & $77.6^{\mathrm{c}}$ & $35.1^{\mathrm{c}}$ & $0.036^{\mathrm{a}}$ & $72.7^{\mathrm{b}}$ & $31.4^{\mathrm{c}}$ & $3,080^{\mathrm{a}}$ \\
\hline 210 & $0.011^{\mathrm{a}}$ & $97.8^{\mathrm{b}}$ & $45.8^{\mathrm{b}}$ & $0.027^{\mathrm{b}}$ & $93.3^{\mathrm{a}}$ & $43.5^{\mathrm{b}}$ & $2,180^{\mathrm{b}}$ \\
\hline 260 & $0.011^{\mathrm{a}}$ & $105^{\mathrm{a}}$ & $62.5^{\mathrm{a}}$ & $0.022^{\mathrm{b}}$ & $99.5^{\mathrm{a}}$ & $49.2^{\mathrm{a}}$ & $1,850^{\mathrm{c}}$ \\
\hline 40 & $0.011^{\mathrm{a}}$ & $85.8^{\mathrm{b}}$ & $40.1^{\mathrm{b}}$ & & & & \\
\hline 50 & $0.011^{\mathrm{a}}$ & $118^{\mathrm{a}}$ & $68.0^{\mathrm{a}}$ & & & & \\
\hline 12 & & & & $0.046^{\mathrm{a}}$ & $76.9^{\mathrm{b}}$ & $34.2^{\mathrm{b}}$ & $2,660^{\mathrm{a}}$ \\
\hline 22 & & & & $0.020^{\mathrm{b}}$ & $96.9^{\mathrm{a}}$ & $46.8^{\mathrm{a}}$ & $2,140^{\mathrm{b}}$ \\
\hline 32 & & & & $0.020^{\mathrm{b}}$ & $91.7^{\mathrm{a}}$ & $43.1^{\mathrm{a}}$ & $2,300^{\mathrm{b}}$ \\
\hline
\end{tabular}

${ }^{\mathrm{a}-\mathrm{c}}$ Means in the same column followed by a different letter are significantly different $(P<0.05)$.

${ }^{1} \mathrm{Dx} 90=90 \%$ of particle diameters were below the stated value; $\mathrm{D}[4,3]=$ volumetric mean.

${ }^{2}$ Interaction effects between solids concentration and inlet temperature were not significant $(P>0.05)$.

2004; Smith et al., 2016a), likely due to their distinct composition. The MPC70 contains more protein and fat. However, lipid oxidation is the main source of offflavors in dried dairy ingredients stored at $21^{\circ} \mathrm{C}$ across a wide range of protein and fat and must be controlled to improve flavor and shelf life (Karagul-Yuceer et al., 2002; Lloyd et al., 2009; Wright et al., 2009; Park et al., 2014; Smith et al., 2016a,b).
Volatile compounds with high odor activity in NFDM and MPC have been identified and confirmed previously (Karagul-Yuceer et al., 2001; Smith et al., 2016a). In NFDM, these compounds include furaneol, butyric acid, methional, o-aminoacetophenone, delta-decalactone, vanillin, hexanoic acid, 2-acetyl-1-pyrolline, octanoic acid, gamma-dodecalactone, 2,4-decadienal, and maltol (Karagul-Yuceer et al., 2001). Many of the same

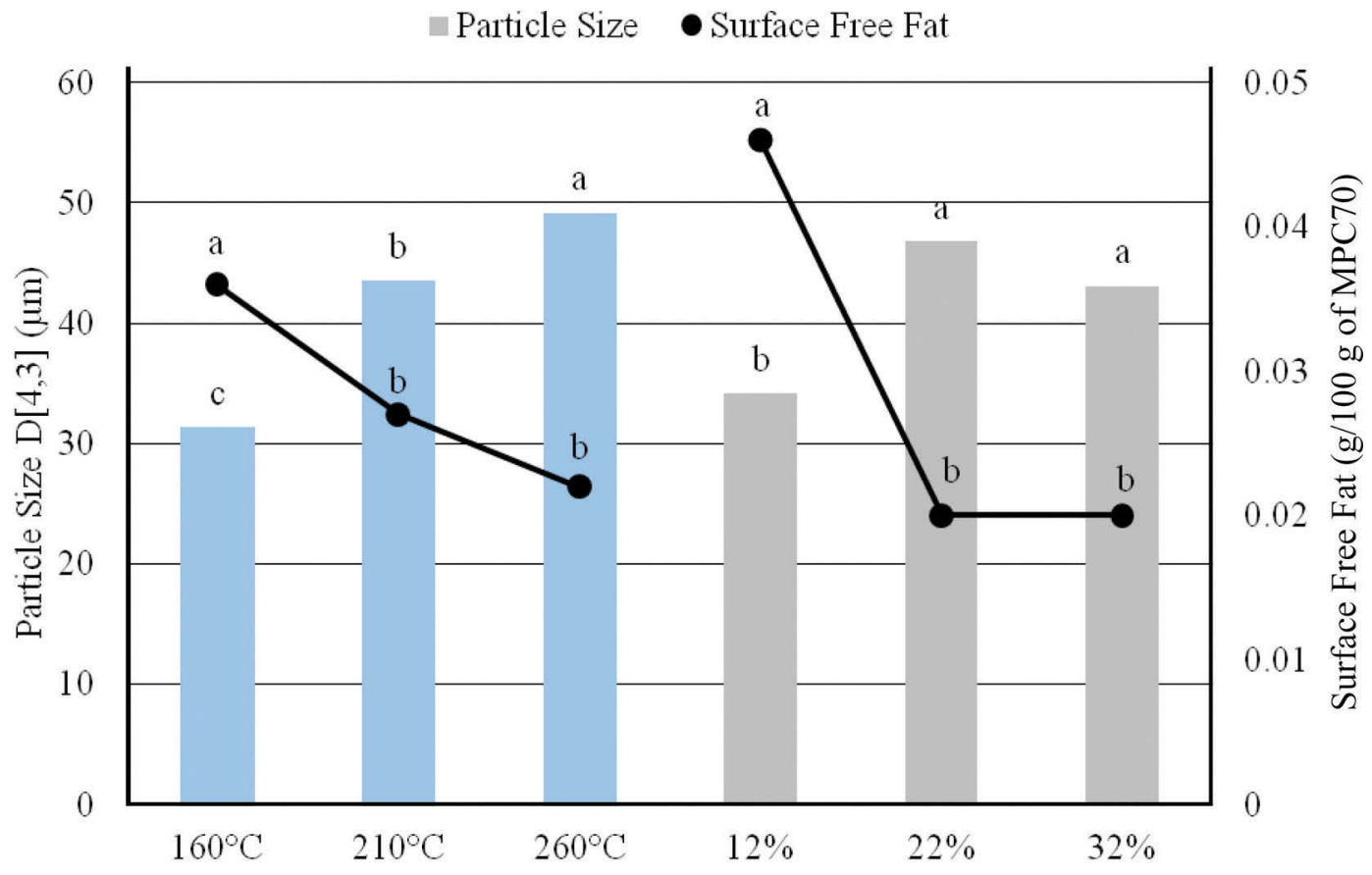

Figure 5. Particle size (gray bars; volumetric mean, D[4,3]) and surface free fat (——; $-100 \mathrm{~g}$ of MPC70) of spray-dried milk protein concentrate $70 \%$ (MPC70). Bars within variable and with different lettering indicate a significant difference $(P<0.05)$. Interaction effects between solids concentration and inlet temperature were not significant $(P>0.05)$. Color version available online. 
compounds were identified with a high odor activity in milk proteins. Those with the highest odor activity were o-aminoacetophenone, nonanal, 1-octen-3-one, dimethyl trisulfide, 2-acetyl-1-pyrolline, heptanal, methional, 1-hexen-3-one, hexanal, dimethyl disulfide, butyric acid, and acetic acid (Smith et al., 2016a).

We identified and quantified many of these compounds in both NFDM and MPC70. The studies mentioned previously used solvent-assisted flavor evaporation to extract the flavor compounds due to the ability to extract compounds of many different classes. We were able to extract many of the same compounds using SSBE, which reduces labor, variability, solvent waste, and increases sample throughput tremendously. Increases in lipid oxidation compounds such as aldehydes, 1-octen-3-one, 2,4-nonadienal, and 2,4-decadienal were observed with increasing cardboard flavor. Smith et al. (2016a) observed increases in these compounds during storage of MPC and they were also correlated with increased cardboard flavor. We also observed that increasing inlet temperature and solids concentration increased sweet aromatic flavor intensity and some associated compounds such as vanillin and 2-acetyl thiazole (Karagul-Yuceer et al., 2001). These increases could be due to generation with the higher heat treatment or a reduction of these compounds by oxidation in samples with increased lipid oxidation.

The fact that increased temperature during spray drying improved flavor may be counterintuitive, but because water is constantly evaporating, the particle temperature does not reach the inlet temperature (Kim et al., 2009). However, we did observe increases in FUR, a marker for Maillard reactions, with elevated inlet temperatures. To our knowledge, FUR has not been reported in dried dairy ingredients that were spray dried under varying conditions. In NFDM, an inlet temperature of $260^{\circ} \mathrm{C}$ increased FUR compared with 160 or $210^{\circ} \mathrm{C}$ and in MPC70 210 and $260^{\circ} \mathrm{C}$ increased FUR compared with $160^{\circ} \mathrm{C}$. This suggests that the heat treatment during spray drying was higher with elevated inlet temperatures. Our results demonstrate that although heat treatment during drying increased with higher inlet temperatures, off-flavors are minimized as observed in the reduction of cardboard flavor by sensory profiling.

Decreased solubility of MPC during storage has been attributed to protein cross-linking with Maillard reaction products (Le et al., 2011a,b). Increases in FUR during storage of MPC have been correlated with decreases in solubility consistent with increased Maillard reaction products (Le et al., 2011a; Smith et al., 2016a). Our results suggest that spray-drying parameters may also play a role in MPC70 solubility through shelf life due to differences in initial FUR content.
Park et al. (2014) observed that lipid oxidation in WPC80 decreased with increasing inlet temperature and feed solids concentration, which is consistent with what we observed for NFDM and MPC70. They hypothesized that it was due to the rate at which the particles dried that influenced flavor. Larger particles are formed when the droplets dry more quickly, which is the case with elevated inlet temperatures and feed solids concentration (Kim et al., 2009). With increased particle size, a decrease occurs in SFF due to a decrease in surface area, which also leads to a decrease in lipid oxidation. In NFDM, we observed differences in particle size with no difference in SFF. This could be because the fat content in NFDM was too low to be able to detect differences in SFF by the method employed. Differences in SFF were detected in MPC70, which had a fat content of more than double that of NFDM $(0.651$ vs. $1.38 \%$ ). Decreases in free fat have been correlated with decreased lipid oxidation and increased shelf life (Keogh et al., 2001; Vega and Roos, 2006; Vignolles et al., 2007; Park et al., 2014).

\section{CONCLUSIONS}

Spray-drying parameters significantly affect the flavor of dried dairy ingredients. Strong consideration should be taken when spray-drying parameters are chosen by ingredient manufacturers to improve the flavor and shelf life of either NFDM or MPC70. Our results also suggest that functionality may be affected and that future work should investigate the relationship between spray-drying parameters and functionality of dried dairy ingredients. Both NFDM and MPC70 were affected by inlet temperature and feed solids concentration during drying. Increasing inlet temperatures and solids concentrations increased sweet aromatic and decreased cardboard flavor and associated volatile lipid oxidation products. We also demonstrated that although the particles do receive a greater heat treatment during drying, flavor quality is improved at elevated inlet temperatures.

\section{ACKNOWLEDGMENTS}

Funding was provided in part by the National Dairy Council (Rosemont, IL). The use of tradenames does not indicate endorsement or indicate a lack of endorsement by those not mentioned.

\section{REFERENCES}

ADPI (American Dairy Products Institute). 2015a. Skim milk powder. Accessed Apr. 30, 2015. https://www.adpi.org/DairyProducts/ DryMilks/SkimMilkPowder/tabid/359/Default.aspx. 
ADPI (American Dairy Products Institute). 2015b. Milk Protein Concentrate. Accessed Nov. 4, 2015. https://www.adpi.org/ DairyProducts/DryMilks/MilkProteinConcentrate/tabid/357/ Default.aspx

AOAC International. 2000. AOAC International Official Methods of Analysis. 17th ed. AOAC Int., Gaithersburg, MD.

Bienvenue, A., R. Jimenez-Flores, and H. Singh. 2003. Rheological properties of concentrated skim milk: Importance of soluble minerals in the changes in viscosity during storage. J. Dairy Sci. $86: 3813-3821$

Cao, J., W. Zhang, S. Wu, C. Liu, Y. Li, H. Li, and L. Zhang. 2015. Short communication: Effects of nanofiltration and evaporation on the physicochemical properties of milk protein during processing of milk protein concentrate. J. Dairy Sci. 98:100-105.

Caudle, A. D., Y. Yoon, and M. A. Drake. 2005. Influence of flavor variability in skim milk powder on consumer acceptability of ingredient applications. J. Food Sci. 70:427-431.

Drake, M. A., Y. Karagul-Yuceer, K. R. Cadwallader, G. V. Civille, and P. S. Tong. 2003. Determination of the sensory attributes of dried milk powders and dairy ingredients. J. Sens. Stud. 18:199216

Drake, M. A., R. E. Miracle, A. D. Caudle, and K. R. Cadwallader 2006. Relating Sensory and Instrumental Analyses. Pages 23-55 in Sensory-Directed Flavor Analysis. R. Marsili, ed. CRC Press, Taylor and Francis Publishing, Boca Raton, FL.

Drake, M. A., R. E. Miracle, and J. M. Wright. 2009. Sensory properties of dairy proteins. Pages 429-448 in Milk Proteins: From Expression to Food. A. Thompson, M. Boland, and H. Singh, ed Elsevier, Amsterdam, the Netherlands.

GEA. 2005. Surface free fat of powder-method 10a. GEA Niro analytical methods. GEA Niro, Soeborg, Denmark.

Isleten, M., and Y. Karagul-Yuceer. 2006. Effects of dried dairy ingredients on physical and sensory properties of nonfat yogurt. J. Dairy Sci. 89:2865-2872.

Karagul-Yuceer, Y., K. R. Cadwallader, and M. A. Drake. 2002. Volatile flavor components of stored nonfat dry milk. J. Agric. Food Chem. 50:305-312.

Karagul-Yuceer, Y., M. A. Drake, and K. R. Cadwallader. 2001. Aroma-active components of nonfat dry milk. J. Agric. Food Chem. 49:2948-2953.

Karagul-Yuceer, Y., M. A. Drake, and K. R. Cadwallader. 2004. Evaluation of the character impact odorants in skim milk powder by sensory studies on model mixtures. J. Sens. Stud. 19:1-13.

Keogh, M. K., B. T. O'Kennedy, J. Kelly, M. A. Auty, P. M. Kelly, A. Fureby, and A. M. Haahr. 2001. Stability to oxidation of spraydried fish oil powder microencapsulated using milk ingredients. J. Food Sci. 66:217-224.

Kim, E. H. J., X. D. Chen, and D. Pearce. 2009. Surface composition of industrial spray-dried milk powders. 2. Effects of spray drying conditions on the surface composition. J. Food Eng. 94:169-181.

Le, T. T., B. Bhandar, J. W. Holland, and H. C. Deeth. 2011b. Maillard reaction and protein cross-linking in relation to the solubility of milk powders. J. Agric. Food Chem. 59:12473-12479.

Le, T. T. B. Bhandari, and H. C. Deeth. 2011a. Chemical and physical changes in milk protein concentrate (MPC80) powder during storage. J. Agric. Food Chem. 59:5465-5473.
Lloyd, M. A., S. J. Hess, and M. A. Drake. 2009. Effect of nitrogen flushing and storage temperature on flavor and shelf-life of whole milk powder. J. Dairy Sci. 92:2409-2422.

Mehta, B. M., and H. C. Deeth. 2015. Blocked lysine in dairy products: Formation, occurrence, analysis, and nutritional implications. Compr. Rev. Food Sci. Food Saf. 15:206-218.

NIST (National Institute of Standards and Technology). 2014. NIST 14 mass spectral library. FarHawk Marketing Services, Evans Mills, NY.

Park, C. W., E. Bastian, B. Farkas, and M. A. Drake. 2014. The effect of feed solids concentration and inlet temperature on the flavor of spray dried whey protein concentrate. J. Food Sci. 79:C19-C24.

Park, C. W., and M. A. Drake. 2014. The distribution of fat in dried dairy particles determines flavor release and flavor stability. J Food Sci. 79:R452-R459.

Prieto, A., O. Basauri, R. Rodil, A. Usobiaga, L. A. Fernandez, N Etxebarria, and O. Zuloaga. 2010. Stir-bar sorptive extraction: A view on method optimization, novel applications, limitations and potential solutions. J. Chromatogr. A 1217:2642-2666.

Resmini, P. L., L. Pellegrino, and G. Battelli. 1990. Accurate quantification of furosine in milk and dairy products by a direct HPLC method. Ital. J. Food Sci. 2:173-183.

Schuck, P. 2013. Dairy protein powders. Pages 3-31 in Advances in Dairy Ingredients. G. W. Smithers and M. A. Augustin, ed. WileyBlackwell, Ames, IA.

Smith, T. J., R. E. Campbell, and M. A. Drake. 2016b. Sensory properties of milk protein ingredients. Pages 197-223 in Advanced Dairy Chemistry Vol. 1B: Proteins: Applied Aspects. P. L. H. McSweeney and J. A. O'Mahony, ed. Springer, New York, NY.

Smith, T. J., R. E. Campbell, Y. Jo, and M. A. Drake. 2016a. Flavor and stability of milk proteins. J. Dairy Sci. 99:4325-4346.

USDA. 2015. Dairy Products Summary. Accessed Apr. 13. 2015. http://usda.mannlib.cornell.edu/usda/current/DairProd/DairProd-04-02-2015.pdf.

Vega, C., and Y. H. Roos. 2006. Invited review: Spray-dried dairy and dairy-like emulsions - Compositional considerations. J. Dairy Sci 89:383-401.

Velez-Ruiz, J. F., and G. V. Barbosa-Canovas. 1998. Rheological properties of concentrated milk as a function of concentration, temperature, and storage time. J. Food Eng. 35:177-190.

Vignolles, M., R. Jeantet, C. Lopez, and P. Schuck. 2007. Free fat, surface fat and dairy powders: Interactions between process and product. A review. Lait 87:187-236.

Vignolles, M., C. Lopez, C. L. Floch-Fouere, J. J. Ehrhardt, S. Mejean, R. Jeantet, and P. Schuck. 2010. Fat supramolecular structure in fat-filled dairy powders: A tool to adjust spray-drying temperatures. Dairy Sci. Technol. 90:287-300.

Wright, B. J., S. E. Zevchak, J. M. Wright, and M. A. Drake. 2009. The impact of agglomeration and storage on flavor and flavor stability of whey protein concentration $80 \%$ and whey protein isolate. J. Food Sci. 74:S17-S29. 